\title{
NEPTUNE'S VISUAL ALBEDO VARIATIONS OVER A SOLAR CYCLE: A PRE-VOYAGER LOOK AT ION-INDUCED NUCLEATION AND CLOUD FORMATION IN NEPTUNE'S TROPOSPHERE
}

\author{
Julianne I. Moses, Mark Allen, and Yuk L. Yung
}

\author{
California Institute of Technology, Pasadena
}

\begin{abstract}
High-energy galactic cosmic rays can penetrate to deep levels within Neptune's atmosphere to form a substantial ionospheric layer in the lower stratosphere and upper troposphere of the planet. Because cosmic-ray modulation in the interplanetary medium creates an inverse relationship between cosmic-ray intensity and solar activity, the ionization rate in the lower atmosphere will vary with the 11-year solar cycle in such a way that maximum ionization will occur at sunspot minimum and minimum ionization at sunspot maximum. This variable ionization may, by the process of ion-induced nucleation, regulate the formation and optical properties of an upper tropospheric haze in the atmosphere of Neptune and could thus provide a mechanism for modulating the planet's visual brightness over a solar cycle. We estimate the rates of cosmic-ray ionization at solar maximum and minimum for a range of proposed Neptune dipole magnetic field strengths; discuss the physics of formation of ion clusters and eventual aerosol particles; and, using a simple radiative transfer model, discuss the ways in which this variable aerosol formation can modulate the geometric albedo of Neptune over a solar cycle. If Voyager spacecraft observations reveal that Neptune's dipole magnetic field is about 1 Gauss or less, then our modeling indicates that variations in an aerosol layer due to differences in cosmic ray ionization may help explain some of the observed brightness variation of Neptune over a solar cycle.
\end{abstract}

\section{Introduction}

Observations carried out since 1972 by Lockwood and Thompson [1986] show that the geometric albedo of Neptune at 472 and $551 \mathrm{~nm}$ varies with an amplitude of about 3\% during the solar cycle. The brightness data anticorrelate with the sunspot number; brightness maximum coincides with solar minimum and brightness minimum with solar maximum. There are at least two types of ideas one can pursue to find an explanation for this interesting solarplanetary phenomenon. One is dubbed the "dark haze theory" and is based on an application of the complex hydrocarbon polymer theory of Allen et al. [1980] to the atmosphere of Neptune [Romani and Atreya, 1988]. In this theory, solar ultraviolet radiation is driving the production of a dark hydrocarbon "smog" at high altitudes on Neptune. A fundamental weakness of this theory is the long timescales required to produce the dark haze; we would expect a phase lag between solar maximum and minimum planetary albedo. Such a phase lag is not observed. This difficulty of the "dark haze theory" provides us impetus to examine another theory. which will be dubbed the "white haze theory."

Ever since Wilson's cloud chamber experiments [Wilson, 1897] it has been known that ions enhance nucleation [e.g. Castleman, 1979]. The possible importance of cosmic-ray ionization in inducing nucleation and forming aerosols in the terrestrial atmosphere has been discussed in the literature [e.g., Ferguson and Amold, 1981]. The "white haze theory" is essentially based on such ideas. We postulate that cosmic-

Copyright 1989 by the American Geophysical Union.

Paper number 89GL03355.

0094-8276/89/89GL-03355\$03.00 ray ionization regulates the formation of an upper tropospheric haze on Neptune, most probably composed of $\mathrm{CH}_{4}$ ice clusters (i.e., a "white haze"). According to various models of the 11-year solar-cycle modulation of galactic cosmic rays (GCR's), convection in the outward-spiralling solar wind and drifts in the large-scale interplanetary magnetic field [Jokipii and Kopriva, 1979] cause cosmic rays to diffuse into the heliosphere less efficiently at solar maximum than at solar minimum. Therefore, the GCR flux will be reduced at solar maximum; fewer cosmic rays will enter the atmosphere of Neptune at that time, ionization and any subsequent aerosol production will thus be reduced, and the planet will appear darker. At solar minimum, the GCR flux will increase again, ultimately leading to more haze production, and a brighter planet. The "white haze" particles are created and lost on short timescales so no solar-cycle phase lag would be expected.

Using a model of Neptune's troposphere and stratosphere, we have estimated the cosmic-ray-induced ionization on Neptune at sunspot maximum and minimum for a range of possible planetary magnetic field strengths. We then assess the impact of this ionization on aerosol formation and the effect of the aerosol layer on the albedo of the planet. The preliminary results presented in this report suggest that the "white haze" theory may, in part, explain Lockwood and Thompson's observations of the variation of Neptune's visible albedo with solar cycle, but further calculations are warranted.

\section{Neutral and Ion Model}

To calculate the ion production rate due to galactic cosmic rays, we have constructed a model of the lower ionosphere of Neptune based on some of the same physical principles that are used in the ionospheric models of Capone and his colleagues [e.g. Capone et al., 1977], except we have used an updated temperature profile and have neglected the secondary particle cascade that results from interactions between primary GCR particles and atmospheric gas molecules. Our neglect of this cascade will cause us to underestimate the ion production rate at levels deeper than about one bar in Neptune's troposphere. The thermal structure adopted in our model is consistent with $\mathrm{K} . \mathrm{H}$ Baines and W.H. Smith [unpublished manuscript, 1989] and is based on the theoretical radiative transfer profile (c) of Appleby [1986] from 10 bar to $10^{-1}$ mbar. A uniform composition with volume mixing ratios $f\left(\mathrm{H}_{2}\right)=0.83, \mathrm{f}(\mathrm{He})$ $=0.15$, and $\mathrm{f}\left(\mathrm{CH}_{4}\right)=0.02$ is assumed for the entire atmosphere below $10^{-1}$ mbar except in the region where we expect methane condensation to occur. In this region, the methane mixing ratio follows a complicated saturation equilibrium curve (see the discussion in the next section). The total absorption cross section for the incident cosmic rays is calculated as in Capone et al. [1977]; specifically, the cross section for absorption of primary cosmic rays by each atmospheric constituent is considered to be independent of energy.

The differential cosmic ray spectra used in our model are similar to those presented by Webber [1987] (see Figure 1). Modifications to Webber's 1 AU spectra were estimated from the cosmic ray gradients measured by Pioneer 10 and 11 and Voyager 1 and 2 that were presented by Webber and 


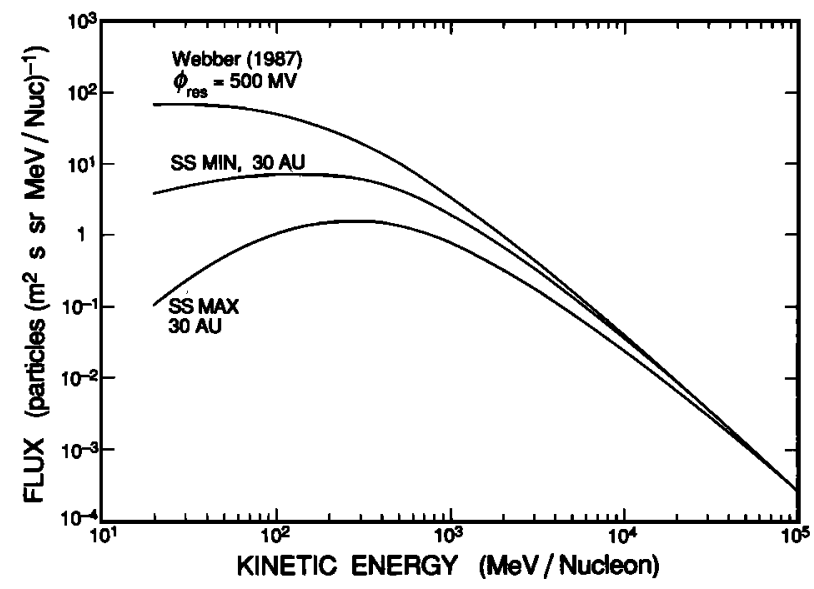

Fig. 1. Differential spectra for galactic cosmic ray particles at $30 \mathrm{AU}$ for sunspot maximum and minimum. The upper curve is an inferred interstellar cosmic ray spectrum for a residual modulation of $500 \mathrm{MV}$ [see Webber, 1987] and is included for scale.

Lockwood [1987]. We assume that the spectra at energies greater than $10^{5} \mathrm{MeV}$ fall off as $E^{-2.75}$. The penetration of charged particles is inhibited by a planetary magnetic field. In particular, a dipole magnetic field will restrict the penetration of all but the most energetic particles to high magnetic latitudes, and GCR ionization effects will be observed only at polar magnetic latitudes on a planet with a strong dipole field.

Once the energy flux of GCR's has been determined, the ion production rate can be simply calculated by a method similar to that of Dubach and Barker [1971]. Table 1 shows the percent increase of the ion production rate at sunspot minimum relative to sunspot maximum at any altitude for different magnetic latitudes and field strengths. For comparison with Capone et al. [1977], the peak ion production rate (at 39 mbar) for a $1 \mathrm{G}$ field at 60 degrees latitude is $23 \mathrm{~cm}^{-3} \mathrm{~s}^{-1}$ and six orders of magnitude smaller at the base of the methane condensation. The important ions produced initially by GCR ionization of neutral $\mathrm{H}_{2}, \mathrm{He}$, and $\mathrm{CH}_{4}$ are $\mathrm{H}_{2}{ }^{+}, \mathrm{He}^{+}, \mathrm{CH}_{4}^{+}, \mathrm{CH}_{3}{ }^{+}$, and $\mathrm{CH}_{2}{ }^{+}$. At the altitudes that we are considering, these ions react rapidly with $\mathrm{H}_{2}$ and $\mathrm{CH}_{4}$ [see Capone et al., 1977 for a more thorough discussion of the ion chemistry] to form a $\mathrm{CH}_{5}{ }^{+}$ion about which neutral $\mathrm{CH}_{4}$ molecules can efficiently cluster.

\section{Ion-Induced Nucleation and Aerosol Production}

The presence of any foreign particles (including ions) in an atmosphere permits effective nucleation and cloud formation by a process known as heterogeneous nucleation. One class of heterogeneous nucleation, ion-induced nucleation, enables aerosol formation to proceed more

Table 1. Difference in Ion-Production Rate between sunspot minimum and maximum (at all altitudes) due to absorption by primary galactic cosmic rays $B$ (Gauss)

$$
70^{\circ} \underset{60^{\circ}}{\text { magnetic latitude }}
$$$$
60^{\circ} \quad 50^{\circ}
$$

$\begin{array}{cccc}0.01 & 40 \% & 40 \% & 40 \% \\ 0.1 & 40 \% & 36 \% & 26 \% \\ 1.0 & 29 \% & 13 \% & 4 \% \\ 10.0 & 5 \% & 0 \% & 0 \%\end{array}$

efficiently for a given saturation ratio $S$ (where $S$ equals the actual equilibrium partial pressure of the condensible species divided by the saturation vapor pressure of the condensible species) than homogeneous nucleation, which is nucleation of vapor onto cluster sites consisting solely of vapor molecules in the absence of foreign particles. This enhancement occurs because ions provide favorable sites for the nucleation process to be initiated. As with any process dealing with phase transformations, the most important parameters that determine the rate at which the transformation proceeds are the temperature of the system and the Gibbs free energy change between the new and old phases. In ion-induced nucleation, the change in free energy between the condensed phase and the pure vapor state is similar to that of homogeneous nucleation [c.f. Castleman, 1979; Seinfeld, 1986], except that there is an additional (negative) term due to the interaction between the central ion of the cluster and the surrounding molecules. This additional term lowers the energy barrier that serves to restrict the rate of formation of large molecular clusters and decreases the critical radius of the cluster - only particles with radii greater than a critical size $R_{\mathrm{p}}$ " can grow spontaneously; smaller clusters are unstable relative to evaporation.

The rate at which clusters of $n$ molecules form about a central ion can be estimated by modifying the formula for the pseudo-steady state homogeneous nucleation rate given by equation (9.44) in Seinfeld [1986]. Our derivation of this equation is similar to Seinfeld's except, in our case, the classical ion formula for the Gibbs free energy [Castleman, 1979] replaces the homogeneous $\Delta G$ and the ion number density replaces the equilibrium monomer concentration. Because $\Delta \mathrm{G}$ depends strongly on the saturation ratio, the ioninduced nucleation rate $J$ increases exponentially with small increases in $S$. For instance, at 875 mbar $(65 \mathrm{~K})$ in Neptune's troposphere, near the base of the condensible methane region, $J=4 \times 10^{-14} \mathrm{~cm}^{-3} \mathrm{~s}^{-1}$ at $S=5.0$ but jumps to $J=6 \mathrm{x}$ $10^{-6} \mathrm{~cm}^{-3} \mathrm{~s}^{-1}$ at $S=5.2$.

In addition, we have found that ion-induced nucleation is much more efficient than homogeneous nucleation at the temperatures in which we are interested; that is, at any particular saturation ratio, the ion-induced nucleation rate is more than one hundred orders of magnitude greater than the homogeneous nucleation rate. Therefore, if no other condensation nuclei are present and if methane is sufficiently supersaturated at low levels in the methane condensation region (i.e. $S \geq 5$ ), then ion-induced nucleation will operate very effectively, and the GCR ion-production rate will provide an easily-reached upper limit to $J$.

Although the rate of production of ion clusters in Neptune's lower atmosphere approaches the GCR ionproduction rate, we cannot immediately convert the production rate into aerosol (or large ion-cluster) number densities. Ions and very small clusters are removed by dissociative recombination; however, large clusters can nucleate rapidly and, once they are formed, can continue to be a source of methane condensation even if the cluster is neutralized by the addition of a free electron. Clusters that exceed the critical size can be lost only by gravitational settling (precipitation), Brownian coagulation, gravitational coalescence, or strong vertical winds. To get some estimate of the abundance and size of these aerosols, we have solved the two time-variable differential equations for particle and methane number densities and have developed a rough steady-state solution. The model consists solely of ioninduced nucleation (with no other condensation nuclei present), condensation, eddy diffusion (to supply $\mathrm{CH}_{4}$ vapor), and particle precipitation. The results (with an eddy diffusion coefficient of $\bar{K} \sim 1 \times 10^{8} \mathrm{~cm}^{2} \mathrm{~s}^{-1}$ in the methane condensation region, derived from mixing-length theory) show that condensation acts rapidly to allow methane gas to cluster about all available ions. The ion clusters quickly 
reach sizes where they can fall out rapidly. We find that a steady-state mass balance between upward diffusion of $\mathrm{CH}_{4}$ molecules and downward precipitation of ice particles exists only if the atmospheric saturation of methane remains just below critical saturation for cluster formation. Thus, the ion cluster formation rate will remain slightly below the GCR ion production rate so that the nucleation can be described as having an "efficiency factor." In our steady-state models, a very small number of very large particles persist at lower levels in the methane condensation region, giving an optical depth in the visible of $0.01<\tau<100$, depending on factors such as the altitude variation of the methane mixing ratio and the efficiency of methane condensation. For instance, in our basic model, we use an efficiency of 0.005 for methane condensation (i.e. methane mixing ratios not quite at critical saturation) to get a steady-state solution of $1.7 \times 10^{-5}$ particles $\mathrm{cm}^{-3}$ of radius $1000 \mu \mathrm{m}$ which persist from 875 mbar to 625 mbar giving an optical depth $\tau \sim 0.7$. Smaller efficiencies give smaller optical depths. The addition of coalescence and, especially, coagulation into the model will serve to reduce the number of particles, the particle lifetimes, and the total optical depth of the haze layer.

To calculate the above steady-state population of aerosols, we have neglected strong vertical winds and convective penetration. Vigorous convective plumes such as those described by Lunine and Hunten [1989] and Stoker and Toon [1989] may be necessary to provide the observed $2 \%$ $\mathrm{CH}_{4}$ mixing ratio in the stratosphere of Neptune. If the total methane mixing ratio (gas plus condensate) is kept constant at $2 \%$ (i.e. by moist convection and rapid vertical winds) then one would expect condensates throughout the possible condensible methane region from about 900 mbar to near the tropopause [Lunine and Hunten, 1989]. In fact, ion-induced nucleation guarantees that nucleation of some kind will occur, and the atmosphere can never be thought of as "clean". Ion clusters will act as condensation nuclei and cause such a large column abundance of particles that the methane cloud will be extremely optically thick. The fact that such a thick methane cloud is not observed over wide areas of the planet [e.g. K.H. Baines and W.H. Smith, unpublished manuscript, 1989] seems to indicate that extreme vertical mixing - convective penetration - is not prevalent on a large areal fraction of the planet. However, convective penetration may still occur in localized areas in sufficiently active plumes to keep the stratospheric mixing ratio at $2 \%$ [e.g. Stoker and Toon, 1989], and we would expect Voyager to see thick white clouds in these regions.

If we assume that convective penetration is not present over wide areas of the planet and if we assume that eddy diffusion is the dominant source of $\mathrm{CH}_{4}$ gas in the nonplume regions, then rapid ion-induced nucleation, condensation, and particle precipitation can limit the vertical extent of the cloud. In this case, the top of the cloud is at levels far below the top of the condensible methane region (i.e. cloud tops at 600 mbar rather than at $100 \mathrm{mbar}$, the tropopause). The situation works as follows. Vertical eddy mixing supplies the methane gas to the condensible region. Very little nucleation and condensation of the $\mathrm{CH}_{4}$ will occur until the saturation ratio reaches values of 5 or so, at which time ion-induced nucleation begins to be effective. Once the critical saturation ratio for ion-induced nucleation is surpassed, critical-sized ion clusters are rapidly formed, and methane will condense efficiently about these clusters. As the gas condenses, methane slowly becomes depleted, the saturation ratio drops to $S=1$, and the whole process can begin again. In our models, we find a methane mixing ratio at each altitude such that the methane density remains in approximate steady state. This methane mixing ratio has the value indicated by a saturation just below critical saturation for ion-induced nucleation of methane at the base of the condensation region but drops gradually to a value defined by $S=1$ at higher altitudes. In the next section, we will show how variations in the methane haze/cloud layer can cause changes in the geometric albedo of a planet.

\section{Aerosol Scattering Model}

K.H. Baines and W.H. Smith [unpublished manuscript, 1989] use ground-based and IUE observations and aerosol models to show that the brightness of Neptune in the visible and near infrared is controlled by a thick cloud deck below 2.7 bar, a thin haze layer around 1 bar, and a thin, dark highaltitude haze at a few mbar. We propose that GCR-induced ionization controls some of the properties of the middle haze layer. In particular, we propose that ion clusters control the cloud particle concentration such that, although atmospheric dynamics controls the cloud locations and vertical extent, ion clusters control the actual number of cloud droplets per unit volume. Thus, if atmospheric motions remain constant over a solar cycle so that the "average" state of the atmosphere does not change significantly, then variation in the optical properties of methane clouds or hazes can be controlled solely by changes in droplet concentration and size. For instance, if the ionization rate is increased while the total methane concentration is held constant, then two radiative consequences result to cause enhanced scattering and a brighter planet: (a) the number of scattering particles is increased, and (b) as the available methane is distributed among more particles, each particle is smaller and stays in the troposphere longer, further increasing the relative number of particles at any one time. These two consequences conspire to make the optical depth vary proportionally with a change in ion cluster production rate; for instance, if the ion production rate is increased by $40 \%$, the optical depth is increased by $40 \%$.

We have modeled the effect of a variable methane haze layer on the geometric albedo of Neptune in the blue (b, 472 nm) using an accurate 8-stream radiative transfer program [Michelangeli et al., unpublished manuscript, 1989]. The calculations were performed for a 2-cloud planet where the bottom cloud level at 2.7 bar is simulated by a surface with Lambert surface reflectivity $r=0.6$. This reflectivity was chosen to keep the geometric albedo of the planet (with a thin haze) near 0.52 at blue wavelengths. Rayleigh scattering was included in the calculations. The upper cloud, or simulated methane haze layer, was modeled with a single scattering albedo of 0.9999 and a varying optical depth. Note that condensed methane has a very low index of refraction at UV and blue wavelengths so should have a large single-scattering albedo in this wavelength region. The actual calculations were performed for isotropic scattering ( $g$ $=0$ ), and we can use van de Hulst and Grossman's [1968] concept of a scaled optical depth $\tau(1-g)$ to compare the result to other scattering asymmetry parameters. We find that the curve of geometric albedo versus haze optical depth follows a sinple "S-shape." Increasing the haze thickness has little effect on the planetary albedo until the haze approaches optical depth unity. Then, small changes in cloud optical thickness cause large variations in planetary albedo until the methane cloud becomes so thick $(\tau-40)$ that further changes have little effect on the cloud albedo.

\section{Conclusions}

Based on the simple modeling in the previous section, it seems that small changes in ion-induced nucleation can result in observable changes in the geometric albedo of the planet as a whole. For instance, if the methane cloud optical depth is in the region from $\tau \sim 1$ to $\tau \sim 40$, and if the cloud optical depth varies by $40 \%$ over a solar cycle (i.e. B $\leq 0.1$ 
G, latitudes $\geq 60^{\circ}$ ) then the planetary albedo will vary by $3 \%$ over this same period, and a $13 \%$ optical depth variation ( $B$ $=1 \mathrm{G}, 60^{\circ}$ latitude) will cause a $1 \%$ variation in planetary albedo. Remember that Lockwood and Thompson's observations showed a $3 \%$ variation in planetary albedo. However, ground-based studies of the aerosol properties of Neptune [e.g. K.H. Baines and W.H. Smith, unpublished manuscript, 1989] indicate that the optical depth of the methane haze layer averaged over the entire planet is $\tau \sim 0.3$ in the blue; the planetary geometric albedo does not change rapidly in this low optical depth region. If we use our basic model of $1.7 \times 10^{-5}$ particles $\mathrm{cm}^{-3}$ of $1000 \mu \mathrm{m}$ radius $(\tau \sim$ 0.7 ) at solar maximum and then, at solar minimum, let the ion production rate increase by $40 \%$, we find that $4.6 \times 10^{-5}$ particles $\mathrm{cm}^{-3}$ of $700 \mu \mathrm{m}$ radius $(\tau \sim 1)$ persist in a steady state model and cause a $\sim 1 \%$ increase in planetary albedo. Therefore, it seems that the "white haze" theory is possibly important but not sufficient to explain completely the observed albedo variations. However, we have not considered the effect of the high altitude hydrocarbon haze. If solar UV is controlling this dark haze layer (by supplying the condensible vapor) then some combination of the "white haze theory" and "dark haze theory" may be acting to explain the observations. It is even possible that ionization by GCR's affects the hydrocarbon aerosol concentrations, but the chemistry of this problem is much more complex than the methane condensate case, and we have left a discussion of the high haze out of the model for now.

At least three constraints on ion-induced nucleation of aerosols on Neptune now need to be discussed. First of all, the atmosphere must be "clean" with no other condensation nuclei present or, at least, the number of ions produced by galactic cosmic rays must exceed the concentration of other condensation nuclei. Heterogeneous condensation onto these nuclei would probably be much more effective than onto small ions. One potential problem on Neptune concems the dark stratospheric haze. Particles from this haze will precipitate down into the methane condensate region. This intrusion of potential condensation nuclei will affect the efficiency of ion-induced nucleation, so simultaneous calculations of condensation and nucleation need to be performed. These calculations will be facilitated by new Voyager data concerning the stratospheric aerosol column densities and gaseous methane abundance. Secondly, the stratosphere must have a continuous source of condensible gas. If the vertical mixing is variable, then the methane haze thickness will be driven by the upwelling rather than the ionization rate, and cloud properties will be controlled by atmospherics dynamics. If thick, convectively-penetrating methane clouds form sporadically on long timescales then an 11-year variable haze layer will be less effective in modulating the planetary albedo. However, as discussed before, convective penetration of methane into Neptune's stratosphere cannot be widespread over a large areal fraction of the planet or ground-based observations would reveal a large methane cloud optical depth. Thirdly, a strong magnetic field will reduce the effectiveness of ion-induced nucleation. A strong (>1 G) field would confine the effects to the magnetic poles and reduce the differences observed between sunspot maximum and minimum.

The ion-induced haze-production process can be important for many reasons besides affecting the long-term brightness of the planet. For example, the presence of a variable haze layer can profoundly affect the dynamics and heat budget in the stratosphere and troposphere. In addition, high-energy solar cosmic rays produced during disturbed solar conditions could noticeably alter the haze thickness for shorter timescales of a few months and introduce weather in the lower stratosphere and troposphere. In all, galactic cosmic ray ionization and corresponding ion-induced aerosol production may be important processes in Neptune's atmosphere and so merit further study.

Acknowledgements. We thank G. W. Lackwood, J. F. Appleby, K. Rages, and D. Crisp for useful discussions. This research was supported in part by NASA grant NAGW 1509 to Califomia Institute of Technology.

\section{References}

Allen, M., J.P. Pinto, and Y.L. Yung, Titan: Aerosol photochemistry and variations related to the sunspot cycle, Astrophys. J. Lett., 242, L125 - L128, 1980.

Appleby, J.F., Radiative-convective equilibrium models of Uranus and Neptune, Icarus, 65, 383 - 405, 1986.

Capone, L.A., R.C. Whitten, S.S. Prasad, and J. Dubach, The ionospheres of Satum, Uranus, and Neptune, Astrophys. J., 215, 977 - 983, 1977.

Castleman, A.W., Jr., Nucleation and molecular clustering about ions, Adv. in Colloid. and Interface Sci., 10, 73 128, 1979.

Dubach, J. and W.A. Barker, Charged particle induced ionization rates in planetary atmospheres, $J$. Atmos. Terr. Phys., 33, 1287 - 1288, 1971.

Ferguson, E.E. and F. Arnold, Ion chemistry of the stratosphere, Accts. Chem. Res., 14, 327 - 334, 1981.

Jokipii, J.R. and D.A. Kopriva, Effects of particle drift on the transport of cosmic rays. III. Numerical models of galactic cosmic-ray modulation, Astrophys. J, 234, 384 392, 1979.

Lockwood, G.W., and D.T. Thompson, Long-term brightness variations of Neptune and the solar cycle modulation of its albedo, Science, 234, 1543 - 1545, 1986.

Lunine, J.I. and D.M. Hunten, Abundance of condensible species at planetary cold traps: The role of moist convection, Planet. Space Sci., 37, 151 - 166, 1989.

Romani, P.N., and S.K. Atreya, Methane photochemistry and haze production on Neptune, Icarus, 74, 424 - 445, 1988.

Seinfeld, J.H., Atmospheric Physics and Chemistry of Air Pollution, John Wiley and Sons, New York, 1986.

Stoker, C.R. and O.B. Toon, Moist convection on Neptune, Geophys. Res. Let., 16, 929 - 932, 1989.

van de Hulst, H.C., and K. Grossman, Multiple light scattering in planetary atmospheres, in The Atmospheres of Venus and Mars, edited by J.C. Brandt and M.B. McElroy, pp. 35 - 55, Gordon and Breach, New York, 1968.

Webber, W.R., The interstellar cosmic ray spectrum and energy density. Interplanetary cosmic ray gradients and a new estimate of the boundary of the heliosphere, Astron. Astrophys., 179, 277 - 284, 1987.

Webber, W.R., and J.A. Lockwood, Interplanetary radial cosmic-ray gradients and their implication for a possible large modulation effect at the heliosphere boundary, Astrophys. J., 317, 534 - 542, 1987.

Wilson, C.T.R., Condensation of water vapor in the presence of dust-free air and other gases, Phil. Trans. R. Soc., AI89, 265 - 274, 1897.

J. Moses, M. Allen, Y. Yung, Caltech 170-25, Pasadena, CA 91125.

(Received: July 21, 1989; revised: October 20, 1989; accepted: October 20, 1989) 